\title{
T cells in COVID-19 - united in diversity
}

\author{
Comprehensive mapping reveals that functional $\mathrm{CD}^{+}$and $\mathrm{CD} 8^{+} \mathrm{T}$ cells targeting multiple regions of SARS-CoV-2 \\ are maintained in the resolution phase of both mild and severe COVID-19, and their magnitude correlates with the \\ antibody response.
}

\section{Leo Swadling and Mala K. Maini}

C $\mathrm{D}^{+}$and $\mathrm{CD}^{+} \mathrm{T}$ cells work with other constituents of a coordinated immune response to first resolve acute viral infections and then to provide protection against reinfection. Careful delineation of the frequency, specificity, functionality and durability of $\mathrm{T}$ cells during COVID-19 is vital to understanding how to use them as biomarkers and targets for immunotherapies or vaccines. In this issue of Nature Immunology, Peng et al..$^{1}$ take a comprehensive approach to characterizing circulating SARS-CoV-2-specific $\mathrm{CD}^{+}$ and $\mathrm{CD}^{+} \mathrm{T}$ cells following resolution of COVID-19. They report a robust and diverse $\mathrm{T}$ cell response targeting multiple structural and non-structural regions of SARS-CoV-2 in most resolved cases, irrespective of whether the individual had mild or severe infection. While the most frequent responses were against peptides spanning spike, membrane and nucleoprotein antigens, all eight regions tested were recognized by multiple individuals, with a maximum of 23 reactive pools in two individuals. Such multispecific $\mathrm{T}$ cell responses are well suited to providing a failsafe form of multilayered protection, mitigating against viral escape by mechanisms such as mutation or variable antigen presentation.

Peng et al. carefully map which parts of the virus are recognized by $\mathrm{T}$ cells using overlapping peptides spanning the whole viral proteome, with the exception of the large ORF-1 region. They use the IFN- $\gamma$-ELISpot assay for initial broad screening of antiviral effector responses, followed by intracellular cytokine staining to show that detected responses comprise polyfunctional $\mathrm{CD}^{+}$and $\mathrm{CD} 8^{+} \mathrm{T}$ cells ${ }^{1}$. Such comprehensive studies are an important first step to identify the targets of SARS-CoV-2-specific T cells so that the heterogeneity of the response can be unpicked and subsequent targeted studies can be carried out. Many factors determine the immunodominance hierarchy of viral antigens, including frequency of naive precursors; the level, timing and location of antigen expression; and the efficiency

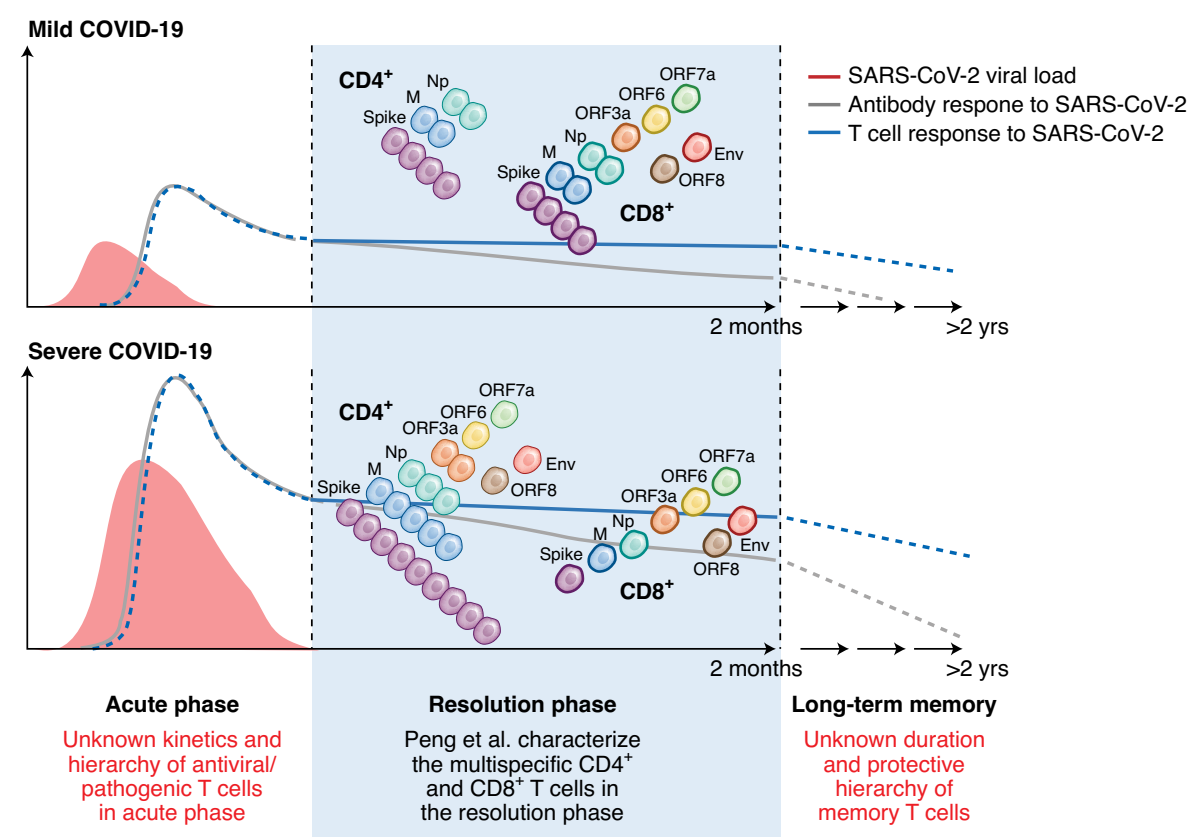

Fig. 1 | Frequency and specificity of T cells in the resolution phase of mild or severe COVID-19. Peng et al. studied the resolution phase of COVID-19 (blue box), showing a broadly targeted CD4 ${ }^{+}$and CD8 ${ }^{+}$ $T$ cell response (cell colors and numbers represent relative frequencies of indicated protein specificities). The total T cell response (solid blue line) is stronger and broader in severe cases (assumed to have had higher viral burden, red curve), correlating with stronger antibody responses (solid gray line). However, there are, proportionally, more CD8 ${ }^{+} \mathrm{T}$ cells in mild disease. Central questions arising from this study (listed in red) concern the unknown hierarchy and kinetics of T cells (dashed blue lines) and antibodies (dashed grey lines) in the acute and memory phases.

of antigen processing and presentation by professional or non-professional antigen presenting cells. The immune response directed against SARS-CoV-2 turns out to be broader than that seen after infection with SARS-CoV-1, wherein T cells are largely specific for spike ${ }^{2}$. A key finding by Peng et al. is that the breadth and magnitude of the $\mathrm{T}$ cell response is greater in those that had more severe COVID-19 (Fig. 1). However, the proportion of the $\mathrm{T}$ cell response that is attributable to $\mathrm{CD}^{+}$ (rather than $\mathrm{CD}^{+}$) $\mathrm{T}$ cells is increased in mild infections, consistent with findings in another study, which showed a higher percentage of activated and proliferating $\mathrm{CD}^{+} \mathrm{T}$ cells in mild as compared to severe COVID-19 ${ }^{1,3}$. These findings hint at a protective role for SARS-CoV-2-specific $\mathrm{CD}^{+} \mathrm{T}$ cells, which is further supported by the greater proportion of clonally expanded $\mathrm{CD}^{+} \mathrm{T}$ cells in the infected lung in mild disease ${ }^{4}$.

The relatively high frequency SARS-CoV-2-specific T cell responses seen after severe COVID-19 are dominated by $\mathrm{CD}^{+} \mathrm{T}$ cells, with responses against spike being particularly abundant (Fig. 1). The correlation Peng et al. observed between spike-specific T cells and antibodies to 
spike (and the receptor binding domain within it) is, therefore, likely attributable to $\mathrm{CD}^{+} \mathrm{T}$ cells, as observed by Grifoni and colleagues $^{5}$. CD4 ${ }^{+} \mathrm{T}$ cells come in many flavors, with follicular helper $\mathrm{T}\left(\mathrm{T}_{\mathrm{FH}}\right)$ cells being crucial for a successful germinal center response generating long-lived plasma cells and broadly neutralizing high-affinity-antibody responses. Studies are now starting to identify the components of a successful T-B cell collaboration in the context of COVID-19 $9^{3,6,7}$. Although Peng et al. did not present viral load data, many other studies have shown viral load is higher in more severe COVID-19 cases. The increase in $\mathrm{CD}^{+} \mathrm{T}$ cells in those with a severe outcome, as also seen with antibodies in a number of studies, is probably a reflection of the increased antigenic burden characteristic of these cases driving stronger immune responses. However, the possibility that stronger $\mathrm{CD} 4^{+} \mathrm{T}$ cell and/or antibody responses contribute to disease severity, rather than just reflecting it, cannot yet be dismissed.

While T cells help to coordinate antiviral immune responses, support the humoral response, limit viral replication and remove infected cells, in doing so they can directly and indirectly contribute to immunopathology. To gain insights into the antiviral versus pathogenic potential of SARS-CoV-2-specific T cells, studies should examine phases that precede the convalescent phase studied here ${ }^{8}$. Longitudinal studies starting at the first stage of acute infection are needed to evaluate whether the timing, magnitude and composition of the early $\mathrm{T}$ cell response is predictive of disease outcome. The temporal evolution of initial immune responses and how they coincide with the exponential viral growth phase can be critical determinants of their efficacy; a delayed or insufficient T cell response could allow uncontrolled viremia to drive subsequent stronger $\mathrm{T}$ cell responses capable of exacerbating tissue damage. Alternatively, the large viral burden triggering strong antibody and $\mathrm{CD} 4^{+} \mathrm{T}$ cell responses in those with more severe disease may predominantly result from a higher viral inoculum or insufficient dampening of early viremia by a failed innate immune response. Dissecting the acute phase response in individuals who clear SARS-CoV-2 infection while remaining asymptomatic has also started to be tackled ${ }^{3}$ and will help define which combination of the myriad immune effectors are best at fighting SARS-CoV-2 without damaging the host.

One reason some individuals may have a head start in the race against the virus is the presence of pre-existing $\mathrm{T}$ cell responses (instigated by a prior coronavirus or other infection) that are able to recognize SARS-CoV-2 and immediately spring into action. Such cross-reactive T cells have been observed in $20-50 \%$ of individuals in some COVID-19 cohorts $^{3,5,9}$ and are being intensively studied to see whether they can mediate any cross-protection, which might contribute to the observed variation in infection severity between individuals, age groups and geographic regions. Peng et al. did not observe SARS-CoV-2-reactive $\mathrm{T}$ cells in the small cohort of healthy controls they studied; this could relate to differences in assay sensitivity, omission of ORF-1 peptides or differences in their cohort's previous exposure to related coronaviruses.

The final pressing set of questions arising from this study concerns the protective potential of the identified multispecific $\mathrm{T}$ cells beyond the convalescent phase. Will particular specificities prove to be more protective or more durable, or will the principle of 'united in diversity' continue to apply? Will some memory cells become 'tissue-resident' populations in the respiratory tract, with the features of longevity and rapid frontline immunosurveillance characteristic of these locally compartmentalized responses? In particular, T cells localized to the airways are critical for protective immunity against related coronaviruses in animal models ${ }^{10}$. There is some controversy arising from recent studies regarding the degree and speed of antibody waning and its relevance in the face of memory $B$ cells, which, if persistent and functional, should replenish the humoral response on virus re-encounter ${ }^{6}$. As with antibodies, those who mounted the strongest and broadest $\mathrm{T}$ cell responses would be predicted to sustain them longer (Fig. 1), but even small, persistent memory populations can rapidly expand on rechallenge. Unlike antibodies, T cells cannot block de novo infection because they only recognize the virus once infected cells present viral peptides, and thus are unlikely to provide sterilizing immunity. But memory T cells can mop up any infected cells that have slipped through a first layer of defense normally provided by antibodies. Thus, $\mathrm{T}$ cell memory at the time of re-exposure may stop severe disease from developing, or it may even lead to aborted subclinical infection. Promisingly, natural SARS-CoV-2 infection in macaques, generating both antibodies and $\mathrm{T}$ cells, does result in protection from rechallenge ${ }^{11}$.

Follow-up studies will be vital to assess the persistence of $\mathrm{T}$ cells directed against different regions of SARS-CoV-2 and how they correlate with memory B cells and neutralizing and non-neutralizing antibodies. T cells generated in response to some human and other animal coronaviruses have proved to be exceptionally long lasting, with responses against SARS-CoV-1, for example, remaining detectable 17 years later ${ }^{9,12}$. The fine epitope mapping carried out by Peng et al. allows the design of HLA-peptide multimers for detailed direct ex vivo $\mathrm{T}$ cell characterization, and initial phenotypic studies have shown a mixed effector and central memory phenotype, as expected from an acute resolving infection ${ }^{1,3}$. Another useful output of this study is the identification of several parts of the virus that are targeted by up to half of the patients tested, despite divergent MHC allele expression. These so-called 'promiscuous' epitopes should be useful for future immunology studies and for consideration in vaccine design. Thus, the data provided by Peng et al. and other recent $\mathrm{T}$ cell studies support the use of vaccine modalities designed to induce both cellular and humoral immunity and raise the possibility of including additional regions beyond spike that have been found to be immunogenic in natural infection.

Leo Swadling and Mala K. Maini (D) $ه$ Division of Infection and Immunity and Institute of Immunity and Transplantation, University College London, London, UK.

$凶_{e-m a i l: m . m a i n i @ u c l . a c . u k}$

Published online: 7 September 2020 https://oi.org/10.1038/s41590-020-0798-y

References

1. Peng, Y. et al. Nat. Immunol. https://doi.org/10.1038/s41590-0200782-6 (2020).

2. Li, C. K. et al. J. Immunol. https://doi.org/10.4049/jimmunol.181. 8.5490 (2008).

3. Sekine, T. et al. Cell https://doi.org/10.1016/j.cell.2020.08.017 (2020).

4. Liao, M. et al. Nat. Med. https://doi.org/10.1038/s41591-0200901-9 (2020).

5. Grifoni, A. et al. Cell https://doi.org/10.1016/j.cell.2020.05.015 (2020).

6. Rodda, L. B. et al. Preprint at medRxiv https://doi.org/10.1101/ 2020.08.11.20171843 (2020).

7. Juno, J. A. et al. Nat. Med. https://doi.org/10.1038/s41591-0200995-0 (2020).

8. Weiskopf, D. et al. Sci. Immunol. https://doi.org/10.1126/ sciimmunol.abd2071 (2020).

9. Le Bert, N. et al. Nature https://doi.org/10.1038/s41586-0202550-z (2020).

10. Zhao, J. et al. Immunity https://doi.org/10.1016/j.immuni.2016. 05.006 (2016).

11. Chandrashekar, A. et al. Science https://doi.org/10.1126/science. abc4776 (2020).

12. Sariol, A. \& Perlman, S. Immunity https://doi.org/10.1016/j. immuni.2020.07.005 (2020).

Competing interests

The authors declare no competing interests. 